\title{
Effects of care transitions intervention mode on the benefit-finding in caregivers for patients with acute cerebral infarction
}

\author{
Xiaoping $\mathrm{Wu}^{1}$, Yueying Gong ${ }^{2, \star}$, Jin $\mathrm{Luo}^{3}$, Xiyan $\mathrm{TaO}^{4}$, Qian Lin ${ }^{5}$, Danping Gao ${ }^{6}$
}

\author{
${ }^{1}$ Department of Neurology, Taizhou \\ Central Hospital, Affiliated Hospital of \\ Taizhou University, 318000 Taizhou, \\ Zhejiang, China \\ ${ }^{2}$ Department of Emergency Intensive \\ Care Unit, Qinghai Provincial People's \\ Hospital, 810007 Xining, Qinghai, China \\ ${ }^{3}$ Department of Operating Room, \\ Taizhou Traditional Chinese Medicine \\ Hospital, 318000 Taizhou, Zhejiang, \\ China \\ ${ }^{4}$ Department of Cardiology, Taizhou \\ Central Hospital, Affiliated Hospital of \\ Taizhou University, 318000 Taizhou, \\ Zhejiang, China \\ ${ }^{5}$ Department of Outpatient, Taizhou \\ Central Hospital, Affiliated Hospital of \\ Taizhou University, 318000 Taizhou, \\ Zhejiang, China \\ ${ }^{6}$ Department of Orthopaedic, Taizhou \\ Central Hospital, Affiliated Hospital of \\ Taizhou University, 318000 Taizhou, \\ Zhejiang, China
}

\section{*Correspondence}

gongyy510@163.com

(Yueying Gong)

\begin{abstract}
To explore the effect of care transitions intervention (CTI) on the sense of benefit-finding of caregivers for patients with acute cerebral infarction (ACI). Ninety caregivers for patients with ACI were divided into two groups according to the random number table method ( $n=45$ in each group). The control group was given regular health guidance, and the intervention group was given care transitions intervention on the basis of the guidance used in the control group. The changes in the sense of benefit-finding and quality of life between the two groups were compared before and after the intervention. There was no statistically significant difference in caregivers between the two groups in gender, age, educational level, occupational status, gender and age of the patients, activities of daily living (ADL) scores before discharge, and the relationships between the caregiver and the patient. Before CTI, there was no statistically significant difference in the caregivers' sense of benefit-finding (including sense of benefit, family relationship, personal growth, social relationship and healthy behavior) and quality of life (including benefit-finding of care, stress of care, choice of care, support to care and money issue) between the two groups. While after CTI, the scores of each dimension of the caregivers' sense of benefitfinding and quality of life in the intervention group were significantly higher than those in the control group $(p<0.05)$. The CTI can help improve the sense of benefit-finding and quality of life of caregivers for patients with ACI.
\end{abstract}

\section{Keywords}

Care transitions intervention; Acute cerebral infarction; Caregiver; Sense of benefitfinding; Quality of life

\section{Introduction}

As one of the fatal diseases endangering human health, acute cerebral infarction (ACI) has the characteristics of high morbidity, high disability and high fatality rate, which brings heavy burdens to the patients' family and the society. The neurological impairments caused by ACI lead to patients' dysfunctions to varying degrees, such as motor dysfunction, dysphagia, etc. Therefore, about $80 \%$ of the cases are taken care by family caregivers for the patients' rehabilitation and daily care after hospital discharge [1].

Systematic evaluations have shown that stroke patients' caregivers have psychological burden to a certain degree during the care process, and also have a sense of benefit-finding [2]. The caregivers' sense of benefit-finding is a positive psychological feeling, referring to the positive growth they experienced and the meanings or benefits they found in stressful and traumatic events. Studies have shown that the sense of benefit-finding can affect an individual's endocrine function, reduce their negative emotions [3], and increase positive experiences [4], thereby improving the quality of care [5]. The sense of benefit-finding of caregivers for ACI patients includes skill improvement, positive attitude, sense of accomplishment, answering and social supports, etc. [6]. However, there is still a lot of room for improvement in levels of caregivers' sense of benefit-finding [7].

\section{Background}

Transitional care [8] refers to a series of measures taken to ensure that patients receive coordinated and continuous health care services due to changes in the diagnosis and treatment environments and care needs during the transfer and transition period. Clinical practice has found that caregivers for acute stroke patients have the greatest caregiving pressure in the first 3 months after discharge [9], especially in the first month. Caregivers have greater demand for care information, serious negative emotions, and relatively weaker caregiving abilities. Therefore, this period of time is an important opportunity to deliver health education or care interventions. The care transitions intervention (CTI) mode can relieve the caregiving pressure and negative emotions of family caregivers for stroke 
patients, and enhance their comprehensive caring abilities, and eventually improve the clinical outcome of the patients $[10,11]$. Based on this, the CTI mode was applied to intervene caregivers for ACI patients in this study, and its effect on the caregivers' sense of benefit-finding was evaluated.

\section{Methods}

\subsection{Research objects}

This study is approved by the TaiZhou Central Hospital Ethics Committee. 90 caregivers for ACI patients who were hospitalized in the Department of Neurology of a " $3 \mathrm{~A}$ " hospital from October 2020 to March 2021, were selected as the research objects. According to the incidence of acute cerebral infarction patients in China and the calculations by G*Power (version 3.1.9.7, University of Dusseldorf, Dusseldorf, Germany), the sample size of this intervention is preliminarily determined to be 90 cases. According to the random number table method, they were divided into the intervention group and the control group ( $n=45$ in each group). Inclusion criteria: (1) Main caregivers for ACI patients in stable condition (the longest average daily care time and caring period $>1$ month); (2) Know the patient's conditions; (3) Voluntarily participate in the study; (4) Age $\geq 18$ years old, clear consciousness, and certain communication skills; (5) No experience of major stress events in the past one month; and (6) Live with the patient after discharge. Exclusion criteria: (1) Have a history of severe anxiety, depression or mental illness; (2) With severe physical illness; (3) Caregivers receiving nursing salary; and (4) Received relevant psychological investigations and interventions recently.

\subsection{Intervention methods}

For the control group, regular health education was provided to patients and their families by the responsible nurse in oneto-one or group basis one week before hospital discharge. The education contents were as follows. (1) Disease knowledge guidance: A health education manual was issued on admission. The content of the manual was mainly diseaserelated knowledge such as risk factors and causes of stroke and ACI, clinical features, treatment and prognosis, as well as basic nursing knowledge such as monitoring of the patient's condition, medication management, dietary guidance, and prevention of complications; (2) Instruction on rehabilitation training methods: explained by the responsible nurse according to the rehabilitation plan, including gait training, balance training, training on the use of assistive tools, etc.; (3) Nursing skill training: based on the patient's daily living ability, caregivers were instructed to master the methods and skills to assist the patients in daily activities such as eating and drinking, dressing and undressing, washing, toileting, and position change; (4) Post-discharge follow-up guidance: the importance of regular review and rehabilitation exercises after discharge was explained, and the brochures and WeChat Public accounts were employed to educate patients and their families.

The intervention group used the CTI mode for intervention on the basis of the conventional health education in the control group. The detailed measures were as follows.

\subsection{Establishment of CTI group}

The CTI group consists of professional doctors and nurses specialising in neurology and rehabilitation, including 1 psychological counselor, 2 responsible nurses, 1 rehabilitation specialist, 1 attending physician, and 2 nursing graduate students. The psychological counselors were responsible for training the group members about the sense of benefit-finding and related psychological knowledge to ensure the effectiveness of the intervention. The responsible nurses were responsible for the simultaneous implementation of the intervention plan. The rehabilitation therapist was responsible for formulating a rehabilitation plan. The attending physician was responsible for the adjustment and risk control of the patient's treatment plan. The graduate students were responsible for coordinating the communication and evaluations of indexes.

\subsection{Determination of the intervention plan}

The intervention framework of this study was based on a review of previous literature and consisted of needs assessment, cognitive evaluation guidance, self-stress and health management, role transition and coping strategy guidance. (1) Needs assessment: On the basis of previous conventional health educations, a self-made "Acute Cerebral Infarction Patient Caregiver Needs Questionnaire" was used to understand needs of caregivers in caring for patients so that they could be given individualised guidance; (2) Cognitive appraisal guidance: caregivers were given targeted guidance to adopt positive cognitive evaluation methods, to discard the idea that negative events would threaten and harm them, and enhance their confidence in overcoming difficulties; (3) Self-stress and health management: Emphasise that improvement of caregivers' physical and mental health is an important guarantee for the quality of care and promotion of patients' recovery, and instruct caregivers on ways to deal with negative emotions and stress; (4) Guidance on role transition and coping strategies: Caregivers were guided to correctly understand their nursing role, encouraged to proactively solve problems through positive communication, and instructed on methods of emotional catharsis and counselling, as well as ways to adjust their mindset, and analyse available social support and health care resources with caregivers.

The entire intervention framework was carried out three days before hospital discharge. On the first day, a needs assessment and cognitive evaluation guidance were conducted. On the second day, self-stress and health management guidance was provided. And on the third day, role transition and coping strategy guidance were carried out. Regular post-discharge follow-up were arranged as follows: weekly telephone followups after discharge and 4-week home visits after discharge. The follow-up visits mainly included: (1) Understanding the patient's rehabilitation status and problems in the nursing care, and providing targeted guidance and assistance; (2) Assessing the psychological condition of the nursing staff and making adjustments; (3) Working with the patient, nursing staff and rehabilitation specialists to develop the next step of rehabilitation and nursing care plan according to the actual situation. 


\subsection{Implementation of intervention}

Prior to the intervention, the attending physician re-assessed the general condition of the patients, and confirmed and verified the information about the patients and their caregivers. (1) Intervention location: Wards of the Department of Neurology; (2) Intervention duration and frequency: once a day for 4560 minutes for three days prior to discharge; (3) Intervention format: One-to-one intervention; (4) Intervention dates: July to December 2020. During the intervention, the environment was quiet and free of other people's interference. The two responsible nurses conducted the intervention simultaneously, seeking the help of a counsellor if necessary. At the end of each intervention, the caregiver's feelings were asked to ensure that they have mastered the content of the intervention and to ensure the effect of the intervention.

\subsection{Evaluation methods}

The general information questionnaire was designed and completed by the research group. The questionnaire includes the gender, age, education level, occupational status of the patients with acute cerebral infarction, as well as the gender, age, ADL scores of the caregivers before discharge, and the relationship between the caregivers and the patients.

The Disease Benefit-finding Scale was revised as described previously [12]. It includes 5 dimensions with 22 items in total, namely acceptance (3 items), family relations (6 items), personal growth (7 items), social relations (3 items), and healthy behaviors (3 items). A 5-point Likert scale was used, with 1 being completely none, and 5 being very much, with a total score of 22 to 110 points. The scale has been tested in caregivers of patients with ischemic stroke [13]. The scale has an overall Cronbach's $\alpha$ coefficient of 0.939 and coefficients of $0.813 \sim 0.976$ for each dimension. The half-reliability was 0.869 , and $0.754 \sim 0.884$ for each dimension. The scale can be used to measure the level of the sense of benefit-finding of caregivers for ACI patients.

The adult caregiver's quality of life questionnaire used was a Chinese version of the questionnaire revised by Mei et al. [14]. It includes 5 dimensions with 31 items in total, which are care benefit-finding (13 items), care pressure ( 6 items), care options (5 items), care support (4 items) and money issue (3 items). The score for each item was 0 (never) to 3 (always), with a total score of 0 to 93 . The higher the score, the better the quality of life. The Cronbach's $\alpha$ coefficient of the questionnaire was 0.924, and the content validity was 0.980. Patients in the two groups were evaluated for general information, disease benefit-finding, and quality of life when they were enrolled, and a second assessment was carried out 4 weeks after hospital discharge.

\subsection{Statistical analysis methods}

IBM SPSS Statistics 25.0 (IBM, Armonk, NY, US) software was used for data analysis. Normally distributed measurement data were expressed as mean \pm standard deviation $(\bar{x} \pm \mathrm{s})$. The comparison between groups was carried out by $t$-test. Non-normally distributed measurement data were expressed by median and interquartile range $M(P 25, P 75)$, and the comparison between groups was carried out by rank sum test. The counting data were expressed as frequency and percentage (\%), and the comparison between groups was carried out by $\chi^{2}$ test. The test standard was $\alpha=0.05$, and the difference with statistical significance was indicated by $p<0.05$.

\section{Results}

\subsection{Comparison of the general information between the two groups}

There was no sample shedding during the whole intervention process. As shown in Table 1, there was no statistically significant difference in caregivers between the two groups in gender, age, educational level, occupational status, gender and age of the patients, ADL scores before discharge, and the relationships between the caregiver and the patient (all $p>$ $0.05)$.

\subsection{Comparison of the caregivers' sense of benefit-finding scores between the two groups before and after CTI}

As shown in Table 2, when the two groups of caregivers were enrolled in the study, there was no statistically significant difference between the two groups in terms of the total score of the sense of benefit-finding and the scores of each dimension (sense of benefit, family relationship, personal growth, social relationship and healthy behavior) (all $p>0.05$ ). After the intervention, the total score of the sense of benefit-finding and the scores of each dimension (sense of benefit, family relationship, personal growth, social relationship and healthy behavior) of the caregivers in the intervention group were significantly higher than those in the control group (all $p<$ $0.05)$.

\subsection{Comparison of the scores in the quality of life between the two groups before and after CTI}

As shown in Table 3, when the two groups of caregivers were enrolled, there was no statistically significant difference in the total scores of the quality of life and the scores of each dimension (benefit-finding of care, stress of care, choice of care, support to care and money issue) between the two groups (all $p>0.05$ ). After the intervention, the total score of the quality of life and the scores of each dimension (benefit-finding of care, stress of care, choice of care, support to care and money issue) in the intervention group were significantly higher than those in the control group (all $p<0.05$ ).

\section{Discussions}

\subsection{The CTI mode can help improve the sense of benefit-finding of the caregivers for ACI patients}

Caregivers of ACI patients assume the primary caregiving tasks after the patients are discharged from the hospital, and there are significant caregiving stresses and demands on caregivers during the transitional period. It has been argued that 
TA B L E 1. Comparison of general information between the two groups.

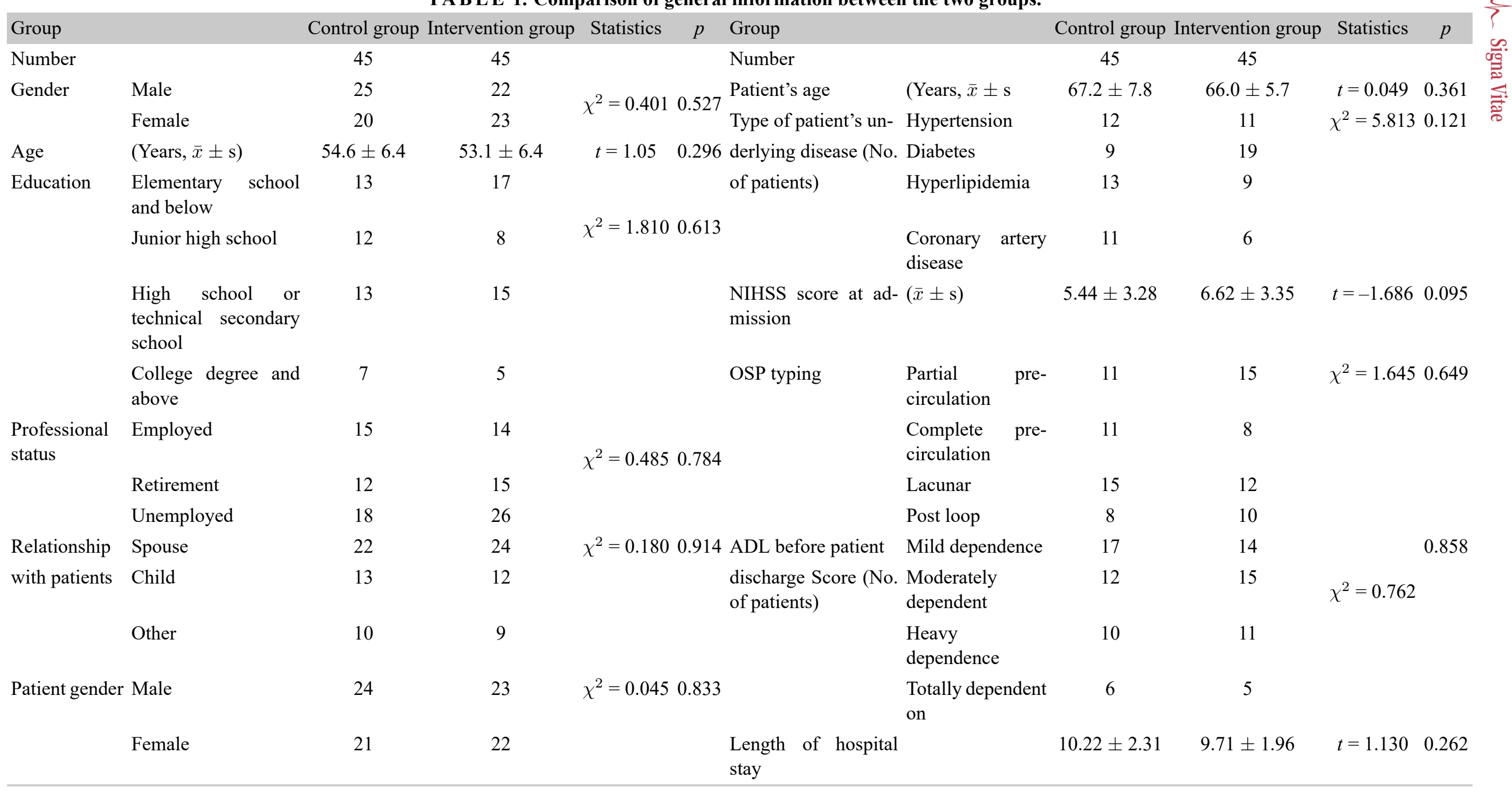


TA B L E 2. Comparison of the caregivers' sense of benefit finding scores between the two groups before and after CTI.

\begin{tabular}{|c|c|c|c|c|c|c|c|c|}
\hline Time & Before & intervention & $t / Z$ & $p$ & After the & intervention & $t / Z$ & $p$ \\
\hline Group & Control group $(n=45)$ & Intervention group $(n=45)$ & & & Control group $(n=45)$ & Intervention group $(n=45)$ & & \\
\hline Total score & $69.5 \pm 5.6$ & $69.4 \pm 4.7$ & 0.082 & 0.935 & $69.3 \pm 5.1$ & $79.0 \pm 1.5$ & -7.894 & $<0.001$ \\
\hline Sense of benefit & $9.0(7.0,11.0)$ & $10.0(8.0,11.0)$ & -1.318 & 0.188 & $10.0(9.0,11.0)$ & $13.0(11.0,14.0)$ & -5.44 & $<0.001$ \\
\hline Family relationship & $20.0(17.0,21.0)$ & $19.0(17.0,20.5)$ & -1.529 & 0.126 & $19.0(17.0,21.0)$ & $20.0(18.0,21.0)$ & -2.12 & 0.034 \\
\hline Personal growth & $20.0(18.0,23.0)$ & $20.0(18.0,23.0)$ & -0.414 & 0.679 & $21.0(19.0,23.0)$ & $24.0(20.5,26.0)$ & -2.997 & 0.003 \\
\hline Social relationship & $9.0(7.5,11.0)$ & $9.0(6.0,10.0)$ & -1.685 & 0.092 & $9.0(7.0,10.0)$ & $12.0(9.5,14.0)$ & -5.89 & $<0.001$ \\
\hline Healthy behavior & $11.0(9.5,13.0)$ & $12.0(10.0,14.0)$ & -1.312 & 0.189 & $11.0(9.0,13.0)$ & $13.0(11.0,13.0)$ & -2.564 & 0.01 \\
\hline
\end{tabular}

T A B L E 3. Comparison in the scores of the quality of life between the two groups before and after CTI.

\begin{tabular}{|c|c|c|c|c|c|c|c|c|}
\hline Time & Before & intervention & $t / Z$ & $p$ & After th & intervention & $t / Z$ & $p$ \\
\hline Group & Control group $(n=45)$ & Intervention group $(n=45)$ & & & Control group $(\mathrm{n}=45)$ & Intervention group $(n=45)$ & & \\
\hline Total score & $40.2 \pm 3.1$ & $39.9 \pm 2.7$ & -0.433 & 0.666 & $41.6 \pm 2.7$ & $52.3 \pm 3.5$ & -17.104 & $<0.001$ \\
\hline Benefit finding of care & $21.0(19.0,22.5)$ & $20.0(19.0,22.0)$ & -1.053 & 0.292 & $21.0(19.0,23.0)$ & $22.0(20.0,24.0)$ & -2.768 & 0.006 \\
\hline Stress of care & $10.0(9.0,12.0)$ & $10.0(9.0,12.0)$ & -0.143 & 0.886 & $10.0(9.0,12.0)$ & $12.0(10.5,14.0)$ & -3.782 & $<0.001$ \\
\hline Choice of care & $3.0(2.0,4.0)$ & $3.0(2.0,4.0)$ & -0.514 & 0.608 & $4.0(3.0,4.0)$ & $7.0(6.0,8.5)$ & -7.967 & $<0.001$ \\
\hline Support to care & $3.0(2.0,4.0)$ & $3.0(2.0,4.0)$ & -0.979 & 0.327 & $3.0(2.0,4.0)$ & $6.0(5.0,7.0)$ & -7.808 & $<0.001$ \\
\hline Money issue & $3.0(2.0,4.0)$ & $3.0(2.5,4.0)$ & -0.267 & 0.789 & $3.0(3.0,4.0)$ & $6.0(5.0,6.0)$ & -7.791 & $<0.001$ \\
\hline
\end{tabular}


[14-16], a positive psychology perspective, tapping into the positive psychology of caregivers can relieve their unhealthy emotions and caregiving pressures, and improve caregiving capacity, thereby improving the patients' quality of life and reducing the risk of re-admission and death. The total score of the sense of benefit-finding of the 90 caregivers for ACI patients in this study was $69.44( \pm 5.11)$, which was lower than that from the study of ischemic stroke caregivers, indicating that the sense of benefit-finding of the caregivers for ACI patients' needs further improvement. This study suggests that the CTI mode is conducive to improving the sense of benefit-finding of caregivers for ACI patients. Yedidia et al. [17]. found that caregivers have the highest demand for self-stress management. This intervention programme guided caregivers to adjust their mentality from four aspects: needs assessment, cognitive evaluation guidance, self-stress and health management, role conversion and coping strategy guidance. In this way, caregivers approach stressful life events with a positive attitude and find benefit from their care, while patients' negative emotions are alleviated and the caregivers' sense of benefit-finding is enhanced.

\subsection{The CTI mode can help improve the quality of life of caregivers for ACI patients}

This study showed that the CTI mode significantly improved the overall score of the caregivers' quality of life and the scores of each dimension (benefit-finding of care, stress of care, choice of care, support to care and money issue). Similarly, Perrin et al. [18] implemented CTI support for stroke caregivers, showing that the caregivers had significantly less care pressures within 3 months after hospital discharge. A number of studies have shown that interventions in the sense of benefit-finding of caregivers are conducive to improving the caregivers' quality of life [19]. Ellis Hill et al. [20] conducted semi-structured interviews with stroke patients and their family members within 1 month after hospital discharge, and found that their needs of professional support were greater. In this study, follow-up visits to the patients and their caregivers were carried out regularly after hospital discharge to provide the caregivers with informational and emotional support to their care work, thereby improving the caregivers' quality of life.

\section{Conclusions}

The transitional period is critical for the rehabilitation of patients with acute cerebral infarction, which requires much higher caring skills for caregivers. This study suggests that the care transitions intervention mode is beneficial to improve the sense of benefit finding and the quality of life of caregivers for acute cerebral infarction patients, thereby further improving the quality of patient caring and patient outcomes. It also suggests that medical staff can carry out care transitions interventions to caregivers for cerebral infarction patients from the perspective of positive psychology, discovering benefits from the caring process, promoting their better adaptation to the role of caregivers, and improving their own caring skills. Since there are many factors influencing the sense of benefit finding of caregivers, follow-up research can combine other psychological indicators to enrich the care transitions intervention mode, which may further enhance the sense of benefit finding of the caregivers and improve the patients' quality of life.

\section{AUTHOR CONTRIBUTIONS}

$\mathrm{XW}$ and YG designed the study, supervised the data collection, $\mathrm{JL}$ analyzed the data, interpreted the data, XT, QL and DG prepared the manuscript for publication and reviewed the draft of the manuscript. All authors have read and approved the manuscript.

\section{ETHICS APPROVAL AND CONSENT TO PARTICIPATE}

Written informed consent was obtained from a legally authorized representative(s) for anonymized patient information to be published in this article. Ethical approval was obtained from the Ethics Committee of TaiZhou Central Hospital (Approval No. 2020-04-20).

\section{ACKNOWLEDGMENT}

Thanks to all the peer reviewers for their opinions and suggestions.

\section{FUNDING}

This research received no external funding.

\section{CONFLICT OF INTEREST}

The authors declare no conflict of interest.

\section{AVAILABILITY OF DATA AND MATERIALS}

All data generated or analyzed during this study are included in this published article.

\section{REFERENCES}

[1] The Lancet Neurology. The shared burden of stroke and dementia. The Lancet Neurology. 2016; 15: 891.

[2] Brainin M, Feigin V, Bath PM, Collantes E, Martins S, Pandian J, et al. Multi-level community interventions for primary stroke prevention: a conceptual approach by the World Stroke Organization. International Journal of Stroke. 2019; 14: 818-825.

[3] Gersdorf R. Benefit-Finding for Dementia Caregivers through Cognitive Reappraisal. The American Journal of Geriatric Psychiatry. 2018; 26 : 404.

[4] Wang AW, Hoyt MA. Benefit finding and diurnal cortisol after prostate cancer: the mediating role of positive affect. Psycho-Oncology. 2018; 27 : 1200-1205.

[5] Brand C, Barry L, Gallagher S. Social support mediates the association between benefit finding and quality of life in caregivers. Journal of Health Psychology. 2016; 21: 1126-1136.

[6] Mei Y, Lin B, Zhang W, Yang D, Wang S, Zhang Z, et al. Benefits finding among Chinese family caregivers of stroke survivors: a qualitative descriptive study. BMJ Open. 2020; 10: e038344. 
[7] Mei Y, Wilson S, Lin B, Li Y, Zhang Z. Benefit finding for Chinese family caregivers of community-dwelling stroke survivors: a cross-sectional study. Journal of Clinical Nursing. 2018; 27: e1419-e1428.

[8] Naylor MD, Aiken LH, Kurtzman ET, Olds DM, Hirschman KB. The Importance of Transitional Care in Achieving Health Reform. Health Affairs. 2011; 30: 746-754.

[9] Ostwald SK, Bernal MP, Cron SG, Godwin KM. Stress Experienced by Stroke Survivors and Spousal Caregivers during the first Year after Discharge from Inpatient Rehabilitation. Topics in Stroke Rehabilitation. 2009; 16: 93-104.

[10] Reeves MJ, Hughes AK, Woodward AT, Freddolino PP, Coursaris $\mathrm{CK}$, Swierenga SJ, et al. Improving transitions in acute stroke patients discharged to home: the Michigan stroke transitions trial (MISTT) protocol. BMC Neurology. 2017; 17: 115.

[11] Liu H, Lou VWQ. Functional recovery of older stroke patients discharged from hospital to home: the effects of cognitive status and different levels of therapy intensity. Journal of Clinical Nursing. 2019; 28: 47-55.

[12] Weaver KE, Llabre MM, Lechner SC, Penedo F, Antoni MH. Comparing unidimensional and multidimensional models of benefit finding in breast and prostate cancer. Quality of Life Research. 2008; 17: 771-781.

[13] Mei Y, Lin B, Li Y, Ding C, Zhang Z. Validity and reliability of Chinese version of Adult Carer Quality of Life questionnaire (AC-QoL) in family caregivers of stroke survivors. PLoS ONE. 2017; 12: e0186680.

[14] Cheng S, Chan WC, Lam LCW. Long-Term Outcomes of the BenefitFinding Group Intervention for Alzheimer Family Caregivers: a ClusterRandomized Double-Blind Controlled Trial. The American Journal of Geriatric Psychiatry. 2019; 27: 984-994.

[15] Hasanali Jafarpoor, Parvaneh Vasli, Houman Manoochehri, Farid Zayeri.
Measuring Family-centered Care in Intensive Care Units: Developing and Testing Psychometric Properties. Signa Vitae. 2020; 16: 82-91.

[16] Najmeh Keshavaraz, Mahin Naderifar, Mohammadreza Firouzkohi, Abdolghani Abdollahimohammad, Majid Reza Akbarizadeh. Effect of Telenursing on the Self-efficacy of Patients with Myocardial Infarction: A Quasi-experimental Study. Signa Vitae. 2020; 16: 92-96.

[17] Yedidia MJ, Tiedemann A. How do family caregivers describe their needs for professional help? The American Journal of Nursing. 2008; 108: 35 37.

[18] Perrin PB, Johnston A, Vogel B, Heesacker M, Vega-Trujillo M, Anderson $\mathrm{J}$, et al. A culturally sensitive Transition Assistance Program for stroke caregivers: examining caregiver mental health and stroke rehabilitation. Journal of Rehabilitation Research and Development. 2010; 47: 605-617.

[19] Gardner MH, Mrug S, Schwebel DC, Phipps S, Whelan K, Madan-Swain A. Benefit Finding and Quality of Life in Caregivers of Childhood Cancer Survivors: The moderating roles of demographic and psychosocial factors. Cancer Nursing. 2017; 40: E28-E37.

[20] Ellis-Hill C, Robison J, Wiles R, McPherson K, Hyndman D, Ashburn A, et al. Going home to get on with life: Patients and carers experiences of being discharged from hospital following a stroke. Disability and Rehabilitation. 2009; 31: 61-72.

How to cite this article: Xiaoping $\mathrm{Wu}$, Yueying Gong, Jin Luo, Xiyan Tao, Qian Lin, Danping Gao. Effects of care transitions intervention mode on the benefit-finding in caregivers for patients with acute cerebral infarction. Signa Vitae. 2021;17(6):112-118. doi:10.22514/sv.2021.224 